\title{
Nonlinear Disturbance Observer-enhanced Depth Control for Submarine: A Sum of Squares Technique
}

\author{
Zhu Zongda ${ }^{1}$; Wu Lifen ${ }^{2}$ \\ Department of Automation \\ Xiamen University \\ Xiamen, China \\ e-mail: ${ }^{1} 1214311412 @ q q . c o m$ (corresponding author); ${ }^{2} 610570939 @ q q . c o m$
}

\begin{abstract}
The state feedback control using sum of squares technique (SOStools) combined with the nonlinear observer is applied to the depth control of a submarine. The mathematical model of the submarine is rewritten as the linear-like form, then the uncertainty caused by the variation of the hydrodynamic coefficients is treated as the disturbance, which can be estimated online by a nonlinear observer. The method can provide good robustness against the nonlinearity as well as the uncertainty, which is verified by the simulation.
\end{abstract}

Keywords-submarine;depth control;nonliniear distlurbance observer;SOS

\section{INTRODUCTION}

The submarine control system is a complex nonlinear system with parameter uncertainties and strong coupling. Large numbers of scholars are participating in this study, and put forward some more fruitful thoughts. Guoliang[1] derived a variable structure controller for submarine. Jian[2] discussed a submarine movement control in vertical plane and derived a robust arithmetic based on mix-sensitivity by studied the state function of submarine linear mode. Shu[3] derived a improved variable structure control method for multiple input-multiple output systems of submarine. The nonlinear disturbance observer achieved good effects in actual system, such as in missiles [5], and aircrafts [6].

This paper proposes a new approach which combines a state feedback controller with a nonlinear disturbance observer. The state feedback controller is formulated using the sum of squares technique (SOStools), and the nonlinear disturbance observer is designed to estimate the nonlinear internal system uncertainty and un-modeled dynamics. The simulation results of submarine in the vertical plane show the controller stability and the observer effectiveness.

\section{STATE FEEDBACK CONTROLLER USING SOSTOOLS}

Consider the system $\dot{x}=f(x)+g(x) u$ written in the linearlike form:

$$
\dot{x}=A(x) x+B(x) u
$$

where $A(x)$ and $B(x)$ are polynomial matrices in $x$.

For the system (1), let $A_{j}(x)$ denotes the $j$-th row of $A(x)$,where $J=\left\{j_{1}, j_{2}, \ldots, j_{m}\right\}$ denote the row indices of
$B(x)$ whose corresponding row is equal to zero, and define $\tilde{x}=\left(x_{j_{1}}, x_{j_{2}}, \ldots, x_{j_{m}}\right)$, then $P(\tilde{x})$ is formulated by $\tilde{x}$.

Our objective is to find a state feedback controller $u(x)=K(x) P^{-1}(x) x$ which renders the equilibrium point $x=0$ stable. Here we present the lemma 1 .

Lemma 1 [4] For a symmetric polynomial matrix $P(x)$ which is nonsingular for all $x$, we have

$$
\frac{\partial P}{\partial x_{i}}(x)=-P(x) \frac{\partial P^{-1}}{\partial x_{i}}(x) P(x)
$$

Lemma 2 [4] For the system (1), suppose exist an $N \times N$ symmetric polynomial matrix $P(\tilde{x})$, an $N \times N$ polynomial matrix $K(x)$, two constants $\varepsilon_{1}>0$ and $\varepsilon_{2}>0$, such that the following sum of squares problem:

$$
\begin{gathered}
v_{1}^{T}\left(P(\tilde{x})-\varepsilon_{1} I\right) v_{1} \\
-v_{2}^{T}\left(A P+P A^{T}+K^{T} B^{T}+B K-\sum_{j \in J} \frac{\partial P}{\partial x_{j}}\left(A_{j} x\right)+\varepsilon_{2} I\right) v_{2}
\end{gathered}
$$

are sums of squares, where $v_{1} \in \mathfrak{R}^{N} \quad v_{2} \in \mathfrak{R}^{M 1}$. Then the state feedback stabilization problem is solvable, and a controller that stabilizes the system is given by

$$
u(x)=K(x) P^{-1}(\tilde{x}) x
$$

Proof: Assume that there exist solutions $P(\tilde{x})$ and $K(x)$ to (3)-(4). Define the function $V(x)$ as follows:

$$
V(x)=x^{T} P^{-1}(\tilde{x}) x
$$

We will show $V(x)$ is a Lyapunov function for the closed-loop system. The time derivate of $V(x)$ is given by

$$
\begin{aligned}
& \frac{d V(x)}{d t}=x^{T}\left[\sum_{j \in J} \frac{\partial P^{-1}}{\partial x_{j}}(\tilde{x})\left(A_{j}(x) x\right)+\left[A(x)+B(x) K(x) P^{-1}(\tilde{x})\right]^{T} P^{-1}(\tilde{x})+(7)\right. \\
& \left.P^{-1}(\tilde{x})\left[A(x)+B(x) K(x) P^{-1}(\tilde{x})\right]\right] x
\end{aligned}
$$

Now, (7) implies that

$$
\begin{aligned}
& -\sum_{j \in J} \frac{\partial P}{\partial x_{j}}(\tilde{x})\left(A_{j}(x) x\right)+P(\tilde{x}) A(x)^{T}+K(x)^{T} B(x)^{T}+ \\
& A(x) P(\tilde{x})+B(x) K(x)+\varepsilon_{2} I<0
\end{aligned}
$$

Multiplying the last expression from the left and right 
by $P(\tilde{x})$, and using the Lemma1. If (4) holds with $\mathcal{E}_{2}>0$ for $x \neq 0$, then $\frac{d V}{d t}(x)$ is negative definite, and therefore, the zero equilibrium is asymptotically stable. Finally, if $P(\tilde{x})$ is a constant matrix, then $V(x)$ is radially unbounded, and the stability holds globally.

\section{EASE NONLINEAR DISTURBANCE OBSERVERS}

In actual application, we should consider the internal system uncertainty and un-modeled dynamics. Consider the system as follows:

$$
\dot{x}=A(x) x+B_{1} d+B_{2}(x) u
$$

For the system (9), the uncertainty caused by the nonlinear internal system and un-modeled dynamics is treated as the disturbance. For all $x$, suppose exist a norm bounded nonsingular matrix $B_{0}$, such that the following expression is equal:

$$
B_{2}(x) B_{0}=B_{1}
$$

So we can get the disturbance observer from the following lemma.

Lemma 3[5] For the system (6), a nonlinear disturbance observer is designed to estimate the disturbances $d$, which are slowly time varying. Given by

$$
\begin{aligned}
& \hat{d}=z+p(x) \\
& \dot{z}=-l(x) B_{1} z-l(x)\left(B_{1} p(x)+A(x) x+B_{2}(x) u\right)
\end{aligned}
$$

where $\hat{d}$ and $z$ are the estimate of the unknown disturbance and internal state of the nonlinear observer, respectively , and $p(x)$ is a nonlinear function to be designed. The nonlinear observer gain $l(x)$ is defined as

$$
l(x)=\frac{\partial p(x)}{\partial x}
$$

If $l(x) B_{1}>0$, the estimation error is global exponential stability for all $x \in \mathfrak{R}^{n}$ :

Proof: The estimation error is defined as

$$
e_{d}=d-\hat{d}
$$

Consider the disturbance $\mathrm{d}$ is slowly time varying ( $\dot{d} \approx 0$ ), then the time derivative of $e_{d}$ is given by

$$
\dot{e}_{d}=\dot{d}-\dot{\hat{d}} \approx 0-\dot{z}-\dot{p}(x)=-\dot{z}-\frac{\partial p(x)}{\partial x} \dot{x}
$$

Submiting (9) and (11), we have

$$
\dot{e}_{d}=l(x) B_{1} z+l(x) B_{1} p(x)-l(x) B_{1} d=-l(x) B_{1} e_{1}
$$

If $l(x) B_{1}>0$, then the estimation error $e_{d}$ is global exponential stability for all $x \in \mathfrak{R}^{n}$.

After the disturbance is estimated by the observer (11), the new closed-loop system controller is given by

$$
u^{*}=u-B_{0} \hat{d}
$$

$$
\dot{x}=A(x) x+B_{1}(d-\hat{d})+B_{2}(x) u
$$

Then the new controller can eliminate the effect of disturbances, and can improve the control performance of closed-loop system.

\section{DEPTH CONTROLLER OF SUBMARINE}

Suppose that only consider the vertical motion of submarine, the other axises motion are neglected. The vertical motion model submarine can be written as follows:

$$
\left\{\begin{array}{l}
\dot{w}=a_{11} s w+a_{12} s q+a_{13} w|w|+a_{14} q|q|+a_{15} \sin \theta+b_{11} s^{2} \delta_{b}+b_{12} s^{2} \delta_{s} \\
\dot{q}=a_{21} s w+a_{22} s q+a_{23} w|w|+a_{24} q|q|+a_{25} \sin \theta+b_{21} s^{2} \delta_{b}+b_{22} s^{2} \delta_{s} \\
\dot{z}=w \cos \theta-s \sin \theta \\
\dot{\theta}=q
\end{array}\right.
$$

where $w$ is heave velocity; $q$ is the pitch rate; $\theta$ is pitch angle; $z$ is submarine depth; $\delta_{b}$ is bow hydroplane angle; $\delta_{s}$ is stern hydroplane angle; the others are kinetic parameters. Let $x=\left[\begin{array}{llll}w & q & z & \theta\end{array}\right]^{T}, u=\left[\begin{array}{ll}\delta_{b} & \delta_{s}\end{array}\right]^{T}$, the system (18) can be rewritten as the linear-like form:

$$
\dot{x}=\left[\begin{array}{cccc}
a_{11} s+a_{13}|w| & a_{12} s+a_{14}|q| & 0 & a_{15} \\
a_{21} s+a_{23}|w| & a_{22} s+a_{24}|q| & 0 & a_{25} \\
1-\frac{1}{2} \theta^{2} & 0 & 0 & s \\
0 & 1 & 0 & 0
\end{array}\right] x+\left[\begin{array}{cc}
b_{11} s^{2} & b_{12} s^{2} \\
b_{21} s^{2} & b_{22} s^{2} \\
0 & 0 \\
0 & 0
\end{array}\right] u
$$

Suppose the desired state is $x_{0}=\left[\begin{array}{llll}0 & 0 & z_{0} & 0\end{array}\right]^{T}$, let $e=x-x_{0}$, the system (18) can be written as the error equation as follows:

$$
\dot{e}=A(x) e+B(x) u
$$

In order to verify the effectiveness of the nonlinear disturbance observer, we design a disturbance observer to estimate the uncertainty caused by the pitch angle velocity state equation . The parameters of the disturbance observer can be chosen as follows:

$$
\begin{aligned}
& l(x)=\left[\begin{array}{llll}
0 & 0.1 & 0 & 0
\end{array}\right] \\
& B_{1}=\left[\begin{array}{llll}
0 & 1 & 0 & 0
\end{array}\right]^{T}
\end{aligned}
$$

$$
B_{0}=\left[\frac{b_{12}}{u^{2}\left(b_{12} b_{21}-b_{11} b_{22}\right)} \frac{-b_{11}}{u^{2}\left(b_{12} b_{21}-b_{11} b_{22}\right)}\right]^{T}
$$

\section{Simulations}

For the error equation (20), we design a state feedback controller using the result stated in lemma 2. The values of $\mathcal{E}_{1}$ and $\mathcal{E}_{2}$ are fixed at 0.001 .

In this paper, our objective is that the submarine drives from $30 \mathrm{~m}$ to $60 \mathrm{~m}$ and moves straight at the certain depth (60m) in the end. The initial state is $x_{0}=\left[\begin{array}{llll}0 & 0 & 30 & 0\end{array}\right]^{T}$, the desired state is $x^{*}=\left[\begin{array}{llll}0 & 0 & 60 & 0\end{array}\right]^{T}$.

Then the system (9) can be written as 


$$
\begin{aligned}
& \delta_{b}=-0.09 w^{2}-0.44 w q-1.35 w z+0.36 w \theta- \\
& 67.15 q^{2}-0.1 q \theta+5.84 q z+0.01 \theta^{2}-0.02 \theta z+ \\
& 0.23 z^{2}+7.9 w-34.05 q+0.69 \theta+1.2 z-5-\hat{\delta}_{b} \\
& \delta_{s}=-0.11 w^{2}-0.90 w q-1.8 w z+1.3 w \theta-35.15 q^{2}- \\
& 0.04 q \theta+5.7 q z+0.02 \theta^{2}-0.04 \theta z+0.53 z^{2}+10.1 w- \\
& 21.05 q+1.2 \theta+1.5 z-10.3-\hat{\delta}_{s}
\end{aligned}
$$

The time history of the depth, the pitch, the bow hydroplane angle, and the stem hydroplane angle are shown from Fig.1 to Fig.4 .From the simulation results, it can be shown that the controller can complete control of the spatial movement of submarine mission, and effectively against the nonlinearity as well as the uncertainty.
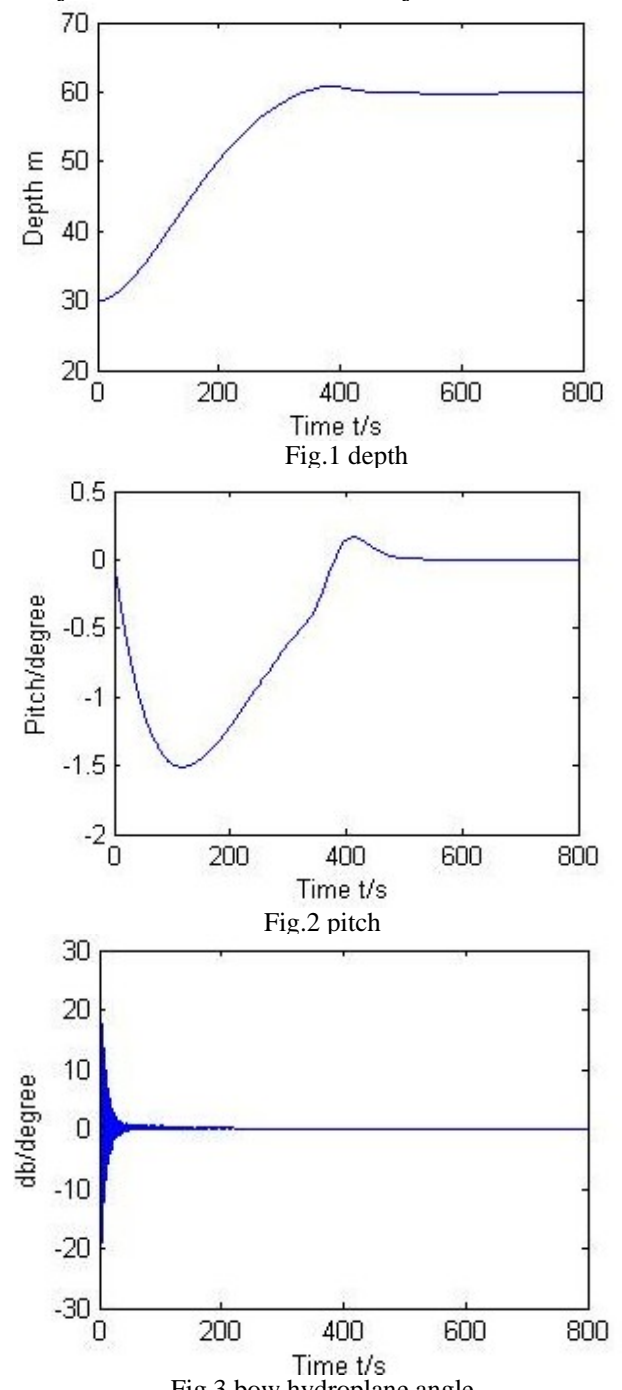

Fig.3 bow hydroplane angle

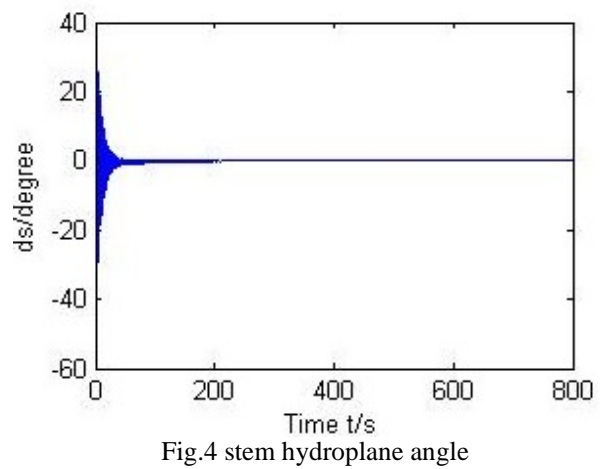

\section{SUMMARY}

According to the simulation in this paper, the state feedback controller combined with the nonlinear disturbance observer has a good control performance against the nonlinearity and the uncertainty. The state feedback control using the sum of squares technique (SOStools) can complete control of the spatial movement of submarine mission. The controller combined with the nonlinear disturbance observer, which can well eliminate the control error which caused by the model uncertainy and satisfactory dynamic behavior.

\section{REFERENCES}

[1] Guoliang Zhao, Luhui Ji and Wang Chong. Sliding Mode Controller for Submarines[J]. Control Theory and Applications,1994,11(3):350355.

[2] Jian Liu, Li Lin, Yiping Guo and Ming Li. Submarinemotion robust control in vertical plane.[J] Ship Science And Technology. 2011, 33(2): 32-36.

[3] Gao Shu, Zhang Xiaoli, Yang Zhiling. Variable structure control method on depth control of submarine[A]. Proceedings of the 30th Chinese Control Conference[C]. 2011: 2593-2596.

[4] S.Prajna, A. Papachristodoulou, and Fen Wu. Nonlinear control synthesis by sum of squares optimization:a Lyapunov-based approach[C].Proc.of the5thAsian Control Conference, 2004:.157-165.

[5] Wenhua Chen. Nonlinear Disturbance Observer-Enhanced Dynamic Inversion Control of Missiles [J]. Journal of Guidance, Control And Dynamics.2003:161-166.

[6] Yuantao Zhang, Weiren Shi and Mingbo Qiu. Sliding Backstepping Control for Fin Stabilizer with Nonlinear Disturbance observer. [J]Control and Decision 2010, 25(8): 1255-1260.

[7] Y. Huang. Nonlinear Optimal Control: An Enhanced Quasi-LPV Approach. PhD thesis, CaliforniaInstitute of Technology, Pasadena, CA, 1999.

[8] S. Prajna, A. Papachristodoulou, and P. A. Pardo. Introducing SOSTOOLS: A general purposesum of squares programming solver. In Proc. IEEE COT\$ on Decision and Control, 2002.

[9] A. Papachristodoulou and S. Prajna . On the construction of Lyapunov functions using the sum of squares decomposition. Decision and Control, 2002 